\title{
Evaluación de la Calidad de la Historia Clínica en el Servicio de Consulta Externa. Unidad Anidada Chunchi, Chimborazo 2018
}

\author{
Evaluation of the Quality of the Clinical History in the External Consultation \\ Service. Nest Unit Chunchi, Chimborazo 2018
}

Andrés Fernando Alcocer Ortega ${ }^{1}$, Mauricio Gustavo Besantes Montoya ${ }^{2} \&$ Lisseth Diana Naranjo Rosas. ${ }^{3}$

\section{Recibido: 10-02-2019 / Revisado: 15-02-209 /Aceptado: 04-03-2019/ Publicado: 27-04-2019}

\begin{abstract}
.
DOI: https://doi.org/10.33262/cienciadigital.v3i2.1.433

Determining as a cornerstone of the entire information system at all levels of health care a quality clinical history, we agree that it is an essential theme in our daily practice, conceptualizing a clinical history appropriately prepared as the written record of all the medical examinations, studies and treatments applied during the course of the disease, the main obtivo of the study was to know the degree of completion of the clinical history in the External Consultation Service of a Unit of First Level of Care, to evaluate areas for improvement .

A transversal descriptive study was carried out, with analysis of the percentage of completion of twenty variables in 147 admissions attended in the outpatient clinic of the Chunchi Anity Unit for 6 months. For data analysis, a database was created in Microsoft Excel 2016.

Of the 20 variables analyzed in the present study it was found that, regarding the quality of the clinical documentation used, the clinical-statistical data and the date of care were present in $100 \%$, family history $(73 \%)$, personal history of interest $(82 \%)$, toxic habits $(8 \%)$, reason for consultation $(95 \%)$, diagnosis, therapeutic orders and vital signs $(100 \%)$.

$96 \%$ of the stories included the evolution sheets. $86 \%$ of the medical records had the post-consultation done by nursing. $59 \%$ of the revised documentation is readable without difficulty and $41 \%$ is legible with some difficulty. $68 \%$ of the medical records had the formulary filled in correctly according to age.
\end{abstract}

${ }^{1}$ Médico General, Ministerio der Salud Publica, Unidad Anida de Chunchi, Ecuador, email: af_andresfernandomed@hotmail.com

${ }^{2}$ Médico General, Ministerio der Salud Publica, Unidad Anida de Chunchi,Ecuador, email: maurigus@hotmail.com

${ }^{3}$ Médico General, Ministerio de Salud Publica, Unidad Anida de Chunchi, Ecuador. email:lisseth14_ever@hotmailcom 
The quality of the registry is variable, acceptable in diagnostic coding, statistical data and date of attention and deficit in personal history, habits, in spite of the high completion (> 95\%) of the reason for consultation and clinical judgment, the relevance of the non-compliance of the documentary filling in $41 \%$ with readable and understandable data.

Keywords: Evaluation, Quality of care. External consultation

\section{Resumen.}

Determinando como piedra angular de todo el sistema de información en todos los niveles de atención de salud a una historia clínica de calidad, concordamos que es un tema esencial en nuestra práctica diaria, conceptualizando a una historia clínica adecuadamente confeccionada como la constancia escrita de todos los exámenes médicos, estudios realizados y tratamientos aplicados durante el transcurso de la enfermedad, el obtuvo principal del estudio fue conocer el grado de cumplimentación de la historia clínica en el Servicio de Consulta Externa de una Unidad de Primer Nivel de Atención, para evaluar áreas de mejora.

Se realizó un Estudio descriptivo transversal, con análisis del porcentaje de cumplimentación de veinte variables en 147 ingresos atendidos en la consulta externa de la Unidad Anidad Chunchi durante 6 meses. Para el análisis de datos se creó una base de datos en Microsoft Excel 2016.

De las 20 variables analizadas en el presente estudio se encontró que, en cuanto a la calidad de la documentación clínica utilizada, los datos clínico-estadísticos y la fecha de atención estaban presentes en el 100\%, antecedentes familiares (73\%), antecedentes personales de interés (82\%), hábitos tóxicos (8\%), motivo de consulta (95\%), el diagnóstico, las órdenes terapéuticas y los signos vitales (100\%).

El 96\% de las historias incluían las hojas de evolución. El 86\% de las historias clínicas tenían realizadas el post consulta por parte de enfermería. El 59\% de la documentación revisada es legible sin dificultad y el $41 \%$ es legible con alguna dificultad. El 68\% de las historias clínicas poseían los formularos llenados de manera correcta de acuerdo con la edad.

La calidad del registro es variable, aceptable en codificación diagnóstica, datos estadísticos y fecha de atención y deficitaria en antecedentes personales, hábitos, a pesar de la alta cumplimentación (>95\%) de motivo de consulta y juicio clínico, se subraya la relevancia del incumplimiento del llenado documentario en un $41 \%$ con datos legibles y comprensibles.

Palabras claves: Evaluación, Historia clínica; Calidad asistencial. Consulta externa 


\section{Introducción.}

El presente artículo surgió del interés por evaluar la calidad de registro de las historias clínicas en un área concurrida de una unidad de primer nivel de atención, como lo constituye la consulta externa de la Unidad Anidada Chunchi.

La evaluación de la calidad de registro de las historias clínicas es parte de las finalidades de la auditoría médica y de vital importancia, debido que las historias clínicas constituyen documentos de alto valor médico, gerencial, legal y académico. Como tal, la información en las mismas contribuye directa e indirectamente en la calidad de atención a los pacientes, optimiza la gestión de los establecimientos, protege los intereses legales del paciente, del personal de salud y del establecimiento; favorece la investigación y docencia.

Las instituciones de salud del sector publico deben garantizar el cumplimiento de sus objetivos y por tanto su misión. Para esto necesita conocer en qué medida se están logrando estos objetivos para así reconocer las fallas de los procesos que impiden prestar un servicio de calidad. Esta evaluación forma parte de uno de los subprocesos de la administración: el control.

Existen diversos métodos utilizados en el proceso de control; uno de ellos es la auditoría médica. Se ha demostrado que la aplicación de estos métodos (entre ellos la auditoría) ha sido beneficiosa en la mejora de la calidad de atención y el desempeño de los médicos.

\section{Marco Teórico}

Como base fundamental se establece en primer lugar a la historia clínica como soporte documental de toda la información que se genera en el acto médico, esta debe reunir una serie de características en cuanto a contenido informativo, ordenación lógica y legibilidad que se hallan recogidas en diversas recomendaciones, normas y legislación al respecto, cumpliendo con cada uno de estos requisitos para ser catalogada como una historia clínica correctamente elaborada.

Existen diversos estudios que han demostrado que la calidad de las historias clínicas influye directamente en la calidad de atención al paciente.

Se ha considerado el buen desarrollo de las historias clínicas como un parámetro para la evaluación de la calidad en la prestación de servicios de salud. Por otra parte, el acto médico que realiza el profesional tiene como sustento a la historia clínica, la misma que tiene que ser veraz y completa. Asimismo, tiene un extraordinario valor jurídico en los casos de responsabilidad médica profesional, al convertirse por orden judicial en la prueba material principal de todos los procesos de responsabilidad profesional médica, constituyendo un documento médico-legal fundamental y de primer orden. 


\section{Metodologia.}

El estudio fue de tipo descriptivo, no experimental, de corte transversal y retrospectivo. El ámbito de investigación comprendió el Servicio de Consulta Externa de la Unidad de Primer Nivel de Atención, Unidad Anidada Chunchi.

El material de estudio estuvo conformado por 147 historias clínicas seleccionadas mediante muestreo aleatorio simple, de entre el total de 2177 ingresos del Servicio de Consulta Externa durante el periodo del 1 de julio al 31 de diciembre de 2018.

Para la estimación del número total de historias, se obtuvo información del Departamento de Archivo de la Clínica.

Se obtuvo un marco muestral total de 2177 historias clínicas, se consideró el máximo tamaño muestra posible basándonos en la fórmula presentada $(\mathrm{N}=\mathrm{Z} 2 * \mathrm{p} * \mathrm{q} * \mathrm{~N} /[\mathrm{e} 2 *(\mathrm{~N}-1)]+[\mathrm{Z} 2$ $\left.\left.{ }^{*} \mathrm{p} * \mathrm{q}\right]\right)=147$ historias clínicas, donde $\mathrm{Z}$ es la confiabilidad al 95\% $(1,96)$, p es 0,5 (asumiendo que 50\% del registro estará llenado correctamente, dado que no contamos con prevalencias obtenidas de estudios similares realizados en entes públicos), q es (1- p), N es el número de historias clínicas totales realizadas en el periodo citado, que corresponde a 2177 historias clínicas y error de precisión (considerado 0,05).

Los criterios de inclusión fueron los todos los pacientes atendidos en el Servicio de consulta externa durante el período de estudio.

Se excluyeron a los pacientes no atendidos en el servicio de consulta externa, historias clínicas de pacientes que estuvieran en procesos médico-legales, historias solicitadas para informe médico u otra auditoria médica, historias clínicas que no se encontraran dentro de los ambientes de archivos clínicos en el momento de la evaluación.

En las historias clínicas de Consulta Externa se consideraron a estudio las variables reflejadas en la Anexo 1, elegidas en base a los estándares marcados para la calificación de la calidad de historia clínica.

Los revisores de las historias clínicas fueron médicos generales rurales del primer nivel de atención de la $\mathrm{UACH}$, por lo que pueden considerarse evaluadores internos respecto al servicio estudiado.

\section{Resultados.}

Durante el periodo de estudio se evaluaron 147 historias clínicas de pacientes atendidos en nuestra unidad de primer nivel de salud. En cuanto a la calidad de la documentación clínica utilizada, los datos clínico-estadísticos y la fecha de atención estaban presentes en el $100 \%$ de los casos. Los antecedentes familiares solo estaban presentes en el $73 \%$ de las historias clínicas revisadas. 
Sin embargo, los antecedentes personales de interés si se recogían en el $82 \%$ de las mismas (figura 1). Se constata la recogida de datos sobre alergias en un $80 \%$ de las historias clínicas, de hábitos tóxicos en un $8 \%$ de las historias. El motivo de consulta 95\%, el diagnóstico, las órdenes terapéuticas y los signos vitales se consignan en $100 \%$.

El 96\% de las historias incluían las hojas de evolución. El 86\% de las historias clínicas tenían realizadas la post consulta por parte de enfermería. El 59\% de la documentación revisada es legible sin dificultad y el $41 \%$ es legible con alguna dificultad. El 68\% de las historias clínicas poseían los formularos llenados de manera correcta de acuerdo con la edad.

Figura 1: Gráfico de cumplimiento de variables

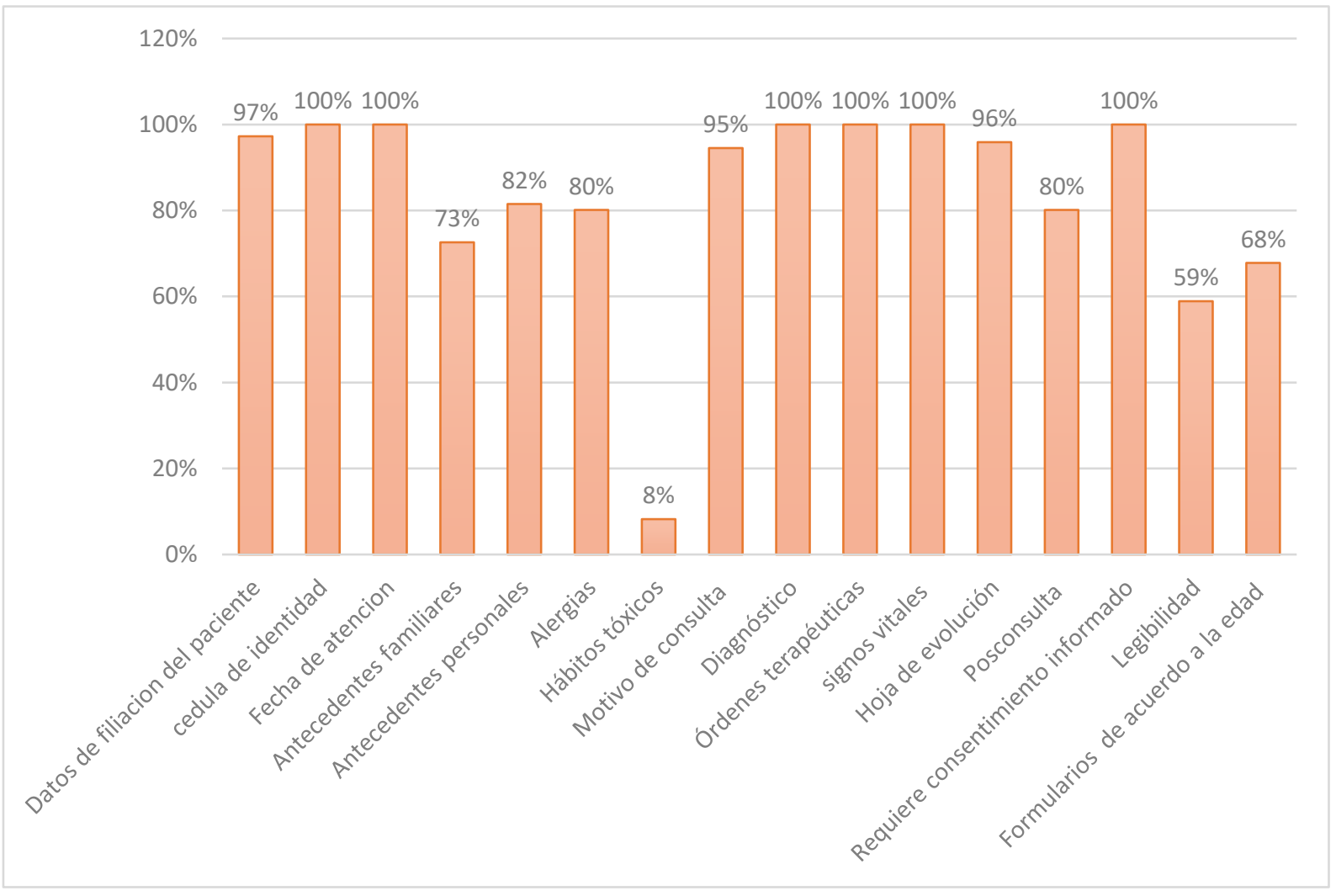

Fuente: Elaboración propia

Conviene destacar que, en los estudios de calidad de la historia clínica, al revisar sus distintos componentes, se trata de objetivar cómo se documenta la asistencia clínica y no la calidad del proceso asistencial. 
De nuestros resultados, cabe resaltar el elevado cumplimiento de las variables registradas de forma informatizada, como los datos de identificación del paciente y la fecha de ingreso. La incorporación de la informática a la gestión de los servicios clínicos se está perfeccionando constantemente y facilita los sistemas de registro y actualización de forma automática. Los antecedentes familiares se recogen en nuestro estudio en un $73 \%$, frente a los antecedentes personales y alergias que son mayormente registrados. Posibles explicaciones para la omisión de los antecedentes familiares son la dificultad del paciente para recodarlos, tratar de eludir la anotación de datos imprecisos o que con frecuencia no se preguntan a la hora de realizar la anamnesis. Asimismo, debería mejorar el registro de hábitos tóxicos como tabaquismo, los cuales se registra en un escaso porcentaje.

Podemos destacar que en las hojas de evolución tanto el motivo de consulta, el diagnóstico, las órdenes terapéuticas los signos vitales se encuentran debidamente registrados en cada una de las historias clínicas, ya que estos parámetros son esenciales para un correcto manejo terapéutico y seguimiento de los pacientes.

Las post consultas de enfermería se encuentran registrado en un porcentaje de un $80 \%$ de las historias clínicas, lo cual da lugar a que todo el proceso de la atención se pueda ver afectado si no se realiza una post consulta adecuada. El consentimiento informado se encontraba adjunto a todas las historias clínicas en las cuales se solicitaba examen de laboratorio para la detección de enfermedades de transmisión sexual, asegurando de esta manera que el paciente este informado sobre los procedimientos a realizarse y las posibles consecuencias de estos.

La legibilidad se pudo evidenciar que solo el $59 \%$ de las historias clínicas no se usaban abreviaturas que den lugar a errores de interpretación y que las indicaciones de estas eran legibles para cualquier personal de la unidad operativa. El correcto uso de los formularios de acuerdo con cada grupo etario se evidencia en menos del $70 \%$ de las historias clínicas valoradas, parámetro en el que los profesionales de salud explican que debido a la falta de tiempo que poseen para atender a cada paciente es muy cortó para el llenado de estos.

Podemos evidenciar que la calidad de la historia clínica es buena, pero existen oportunidades de mejora en la cumplimentación de antecedentes familiares, dejar constancia escrita de la presencia o ausencia de hábitos tóxicos (tabaco). Mejorar la caligrafía y evitar el uso de abreviaturas en las historias clínicas y evaluar si el tiempo establecido por norma en la atención de los pacientes es el suficiente para el llenado de todos los formularios correspondientes a cada grupo etario. 


\section{Conclusiones.}

En conjunto del análisis de las variables se registró un elevado cumplimiento de forma informatizada, como los datos de identificación del paciente y la fecha de ingreso, catalogando como una Historia Clínica: Buena.

Se determinó una omisión de los antecedentes familiares con tres frecuentes escenarios: dificultad del paciente para recodarlos, tratar de eludir la anotación de datos imprecisos o no se preguntan a la hora de realizar la anamnesis.

Se estableció un su registro de hábitos tóxicos, variable de importancia en el diagnóstico de Enfermedades crónicas no transmisibles.

El motivo de consulta, el diagnóstico, las órdenes terapéuticas, así como los signos vitales se encuentran debidamente registrados en cada una de las historias clínicas (100\%), parámetro fundamental para un correcto manejo terapéutico y seguimiento de los pacientes

El consentimiento informado se encontraba adjunto a todas las historias clínicas, garantizando los principios Bioéticos.

La legibilidad se encontró afectada en $41 \%$ de las historias clínicas lo cual puede llevar a errores de interpretación y posibles repercusiones en la salud de los pacientes.

\section{Referencias bibliográficas.}

Stoner J, Freeman R, Gilbert D. Administración. 6ta Edición. México. 1996.

Scales D, Dainty K, Hales B, Pinto R, Fowler RA, Adhikari NK, Zwarenstein M. A multifaceted intervention for quality improvement in a network of intensive care units: a cluster randomized trial. JAMA. 2011 Jam 26; 305(4):363-72. doi: 10.1001/ jama.2010

Vodstrcil LA, Hocking JS, Cummings R, Chen MY, Bradshaw CS, Read TR, Sze JK, Fairley CK. Computer assisted self interviewing in a sexual health clinic as part of routine clinical care; impact on service and patient and clinician views. PloS One. 2011 Mar;6(3):1-9. doi: 10.1371/journal. pone.0018456

Hartig JR, Allison J. Physician performance impro- vement: an overview of methodologies. Clin Exp Rheumatol. 2007 Nov-Dec; 25(6 Suppl 47):50-4

Ley 41/2002, de 14 de noviembre, básica reguladora de la autonomía del paciente y de derechos y obligaciones en materia de información y documentación clínica. BOE num 274, pp. 40126-40132.2000 
Carpintero JM, Ochoa FJ, Acítores JM, Ferrús JA, Fernández JA. Grado de cumplimentación de la Historia Clínica de Urgencias como indicadores de la Calidad Asistencial. Emergencias. 1997; 9 (1): 26 - 30

Rocano EP. Evaluación de la calidad de los registros de las historias clínicas de los pacientes fallecidos en el Servicio de Emergencia de un Hospital General. Rev Soc Peru Med Int. 2008;21(2):51-4

Bocanegra S, Bocanegra G; Alvarado V. Nivel de calidad del registro de las historias clínicas de pacientes apendicectomizados en el Hospital Belén de Trujillo de enero a junio de 2007. Rev Med Vallejiana. 2008;5(2):115 -24.

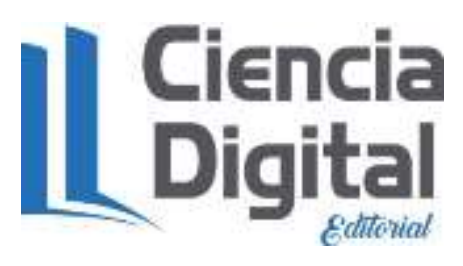




\section{PARA CITAR EL ARTÍCULO INDEXADO.}

Alcocer Ortega, A., Besantes Montoya, M., \& Naranjo Rosas, L. (2019). Evaluación de la calidad de la historia clínica en el servicio de consulta externa. Unidad Anidada Chunchi, Chimborazo 2018.

Ciencia Digital, 3(2.1), 85-92. https://doi.org/10.33262/cienciadigital.v3i2.1.433

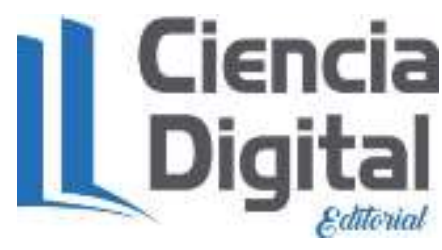

El artículo que se publica es de exclusiva responsabilidad de los autores y no necesariamente reflejan el pensamiento de la Revista Ciencia Digital.

El artículo queda en propiedad de la revista y, por tanto, su publicación parcial y/o total en otro medio tiene que ser autorizado por el director de la Revista Ciencia Digital.
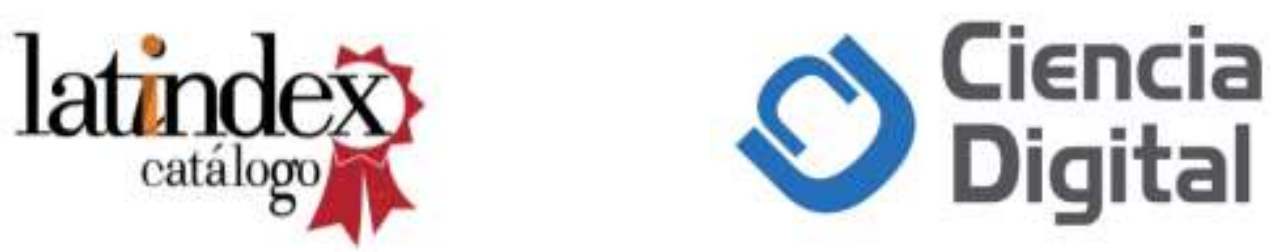